\title{
Effect of spiritual care on the care burden of families of children with cancer: a randomized controlled trial
}

\author{
MINOO ASADZANDI ${ }^{1-3, A, E, ~ F, ~ S A F O R A ~ S H A H R A B I ~ F A R A H A N Y 4, ~ B, ~ c, ~}$ \\ ORCID ID: 0000-0001-5149-6374 \\ HASSAN ABOLGHASEMY3, 5, 6, D, MOHSEN SABERII, , ABASS EBADI
}

${ }^{1}$ Medicine, Quran and Hadith Research Center, Baqiyatallah University of Medical Sciences, Tehran, Iran

${ }^{2}$ Spiritual Health Research Center, Qom University of Medical Sciences, Qom, Iran

${ }^{3}$ Department of Spiritual Health, Academy of Medical Sciences, Tehran, Iran

${ }^{4}$ Faculty of Nursing, Baqiyatallah University of Medical Sciences, Tehran, Iran

${ }^{5}$ Hereditary Diseases of Blood Research Center, Shaheed Beheshti University of Medical Sciences, Tehran, Iran

${ }^{6}$ Department of Pediatrics, Faculty of Medicine, Baqiyatallah University of Medical Sciences, Tehran, Iran

${ }^{7}$ Behavioral Sciences Research Center, Life Style Institute, Nursing Faculty, Baqiyatallah University of Medical

Sciences, Tehran, Iran

A - Study Design, B - Data Collection, C - Statistical Analysis, D - Data Interpretation, E - Manuscript Preparation, F - Literature Search, G - Funds Collection

Summary Background. Cancer creates a crisis for families. In children oncology, parental care is an important priority of the home-care approach. Parents' education is essential for the continuance of child care.

Objectives. This study was conducted to determine the effect of spiritual care based on the Sound Heart Model on the care burden of families of children with cancer.

Material and methods. In a randomized controlled trial, 72 families of children with cancer were selected according to inclusion criteria. In the educational support system, spiritual care based on the Sound Heart Model was carries out. Training spiritual skills was conducted through virtual education within the social network, along with the donation of educational software. The purpose of spiritual care was: creating optimism, hope and courage to face the disease through faith therapy, spiritual self-awareness and development of social communication by forgiveness training, using the blessings of nature. The development of relationships with God, self people and nature was advised. The care burden questionnaire was completed by parents in the intervention and control groups before and immediately after intervention. The data was analyzed.

Results. There was no significant difference between the level of parents' care burden before intervention in the two groups $(p=0.523)$. After intervention, the care burden of the intervention group was significantly decreased compared to the control group $(p<0.001)$, and there was no significant change in the control group $(p=0.415)$.

Conclusions. This study showed that spiritual care decreased the level of parents' care burden. The use of the model to meet the spiritual needs of the family of children with cancer is suggested.

Key words: neoplasms, nursing, patient education, spitrituality.

Asadzandi M, Farahany SS, Abolghasemy H, Saberi M, Ebadi A. Effect of spiritual care on the care burden of families of children with cancer: a randomized controlled trial. Fam Med Prim Care Rev 2021; 23(3): 279-283, doi: https://doi.org/10.5114/ fmpcr.2021.108189.

\section{Background}

Due to the increasing growth of cancer and its psycho-socio-spiritual conflicts, research on this phenomenon is highly regarded by countries such as Iran [1]. A survey of the 10 most common cancers, their prevalence factors, patients' awareness and the impact of self-care behavior on cancer prevention and control were the priority of the Deputy of Health and Medical Education in the Armed Forces in Iran [2]. The number of children with cancer in Iran in 2018 was reported between 4,500$-6,000$ individuals, 1,100 of them were belonged to families of the armed forces. Cancer is the second leading cause of death in children [3]. Leukemia, brain tumors and lymphoma are the most common pediatric cancers, which entails long-term and costly treatment and imposes a care burden on the family [4].

Parents of children with cancer tend to take care of their children at home [5], but lack of knowledge about the cause of the disease, treatment and care methods, unpredictability of cancer prognosis, long-term care, frequent hospitalization and economic consequences, separation from the child during hospitalization, suffering imposed on the child during illness, changes in the child's physical condition and reactions of the community, are factors that cause family suffering and leads to psycho-socio-spiritual conflicts that have adverse effects on the child [6]. Although the home care approach is noticeable in Iran due to its social and economic benefits to the patient, family and health system and, at present, more than $90 \%$ of cancer care is done at home, and parental care is an important priority in child oncology [7], but taking care of a child with cancer creates changes in the lifestyle and roles of parents [8]. Parents as primary caregivers should report any related complications and the child's condition to the treatment team. They play a key role in managing various aspects of the symptoms of the illness, creating adaptation in the child, pursuing therapeutic measures and adapting to these responsibilities [9]. In recent years, atten- 
tion has been paid to spiritual care in scientific communities due to parents' care burden, which increases with the severity of the child's illness [10].

Care burden is not considered a disease, but is the cause of the caregivers' suffering which is defined, it is defined as the bio-psycho-social response of caregivers due to an imbalance between patient care needs, financial resources, caregivers' social roles and their physical and mental status [11]. Care burden causes family isolation, lack of hope for social support, family disruption and the inability to perform daily life activities, inadequate patient care and eventually abandonment of the patient [12]. Caregivers will not have the ability of adaptation to the high levels of care burden with little social support and lack of home care services [13]. This situation impose extra burdens and costs on the families. Implementing spiritual care and family empowerment can facilitate home care [14].

Empowerment as a participatory education approach requires looking at the needs of families of children with cancer, including: expressing the meaning of the illness, the cause of the child's suffering, teaching ways to reduce "separation anxiety", "fear of death" and familiarity with the cause of the disease, treatment and care methods [15]. It should be applied with family participation in designing a community-based care plan in line with the culture, values and preferences of the family members [16].

In Iran, $98 \%$ of people are Muslim, and $90 \%$ are Shiite. Religious culture is blended with the Muslim lifestyle, and religious beliefs play an important role in adaptation to critical situations [17]. Spirituality and religion are not separated in Islam, and Islamic rituals shape spirituality based on faith and good deeds [18] that enable people to overcome life-threatening difficulties, move with the flow of life and return to their initial condition in a short period [19].

The spiritual care model of the Sound Heart, in line with the Muslims belief in "the soul" and "life after death", introduces spiritual health as having a "sound heart" and life events as a "divine experiment", which can be managed in the light of love and power of God as a healer [20]. The model introduces the fear and anxiety of the future and the regret of losing health as the most important spiritual distress, arising from despair of God's mercy. In the spiritual care program, by developing a relationship with God, the patient and family are helped to "develop courage" in the face of life's problems, creating positive thinking, hope and optimism [21].

\section{Objectives}

This study was conducted to determine the effect of spiritual care based on the Sound Heart Model on the care burden of parents of children with cancer.

\section{Material and methods}

This semi-experimental study with pre- and post-test design was conducted in 2018 on 72 parents of children with cancer from armed forces families. Families that referred to the Baqiyatallah hospital and the Children's Medical Center who were willing to participate were formed research samples.

\section{Sample size determination}

The sample size was calculated as 36 families for each group by using the mean and standard deviation obtained from a similar study (with $\alpha=5 \%$ and power $=90 \%$ ) [22] and Altman's Nomogram, with a $20 \%$ loss. They were selected according to the inclusion criteria: being able to communicate and complete the questionnaire, being familiar with the Persian language, without a history of: addiction, chronic mental illness, taking psychiatric drugs, participating in similar research, having other critically ill children.

\section{Control and intervention groups}

In a meeting with the investigator, after explaining the research objectives and emphasizing on the confidentiality of any personal information that would lead to disclosure of parents' identity and obtaining informed consent, the samples completed the questionnaires. They were then randomly assigned to test and control groups before intervention by forming quadruple blocks. The intervention was performed for the test group through donated educational software along with once-a-week communication through the social network. The control group only received routine care. At the end of the intervention, the questionnaires were completed by both groups, and the results were analyzed. The educational software was also donated to the control group, and their questions were answered.

\section{Intervention program}

In the educational support system, spiritual care was performed according to the algorithm of the Sound Heart Model [21] through virtual education within the social network and the donation of educational software. The training software included: 16 PowerPoint presentations, the hand book file: "Spiritual Care Guide for Patients' Families" [23], educational clips, animation about cancer and its treatments with child-friendly language, religious poetry and wallpapers of nature with bird song. The content validity of the software was confirmed by the professors of the Blood and Cancer Research Center of Shahid Beheshti University located in Mofid Hospital in Tehran. Virtual education witin the social network was followed by 16 spiritual counseling sessions, 20-30 minutes once a week, with emphasis on improving the four-person relationships with God, self, people and nature (according to Table 1). The purpose of spiritual care was: creating optimism, hope and courage to face the disease, spiritual self-awareness, development of social communication with forgiveness training and using the blessings of nature.

\section{Table 1. Spiritual titles for parents' education}

1 Cancer, its causes and methods of treatment and care, ways of coping with the disease

2 Home care problems, care burden, its causes and consequences 3 Spiritual empowerment as a strategy to reduce care burden

44 Empowerment factors: knowledge and skills, trust in God, development of communication, strengthening motivation

5 Definition of spirituality, positive impact of spirituality on health

$6 \quad$ Spiritual health as having a sound heart, effect of spiritual health on other dimensions of health

7 Characteristics of a person with spiritual health

8 When do we need spiritual care? Who are the providers of spiritual care? Parents as spiritual counselors

9 Despair of God's mercy as an important cause of spiritual distresses

10 The disease as a divine test, the philosophy of human testing, strategies for coping with stress (patience, piety and faith, submission to God's will, attention to the presence of God)

11 Creating the courage to face the disease through faith-therapy (remembrance of God, prayer, hope, positive thinking)

12 Faith therapy, effects of prayer, touch with healing prayers, Islamic ritual of sleep

13 Spiritual self-care, the need to pay attention to spiritual distress, types of spiritual distress

14 Spiritual self-awareness, spiritual care guidelines based on religious evidence

15 Ways to communicate with others based on generosity, pardon another's mistakes and kindness

16 Use of nature blessings, motivation and motivational enhancement methods 


\section{Instruments}

Data was collected by:

1 - Demographic questionnaire (12 questions), including two parts: A: Caregiver information, such as: age, gender, marriage status, education, family relationship and illness; B: Patient information, such as: duration of illness, type of cancer, ability to perform personal tasks, the amount of care needs.

2 - Care burden questionnaire (CBI) with 24 items, invented by Novak and Guest in 1989. The questionnaire consists of 5 subscales: 1 - Time dependent care burden (indicates the daily amount of time that a caregiver takes care of the patient) with 5 items ( 1 to 5 ); 2 - Evolutionary care burden (the pressure on the caregivers due to the developmental needs of ill child) with 5 items (6 to 10); 3 - Physical care burden (physical exhaustion during patient care) with 4 items (11 to 14 ); 4 -Social care burden (which affects the social aspects of a caregiver's life while taking care of a sick person) with 5 items (15-19); 5 - Emotional care burden (which affects the emotions and feelings of the caregiver) with 5 items (20 to 24). This questionnaire has good reliability. Cronbach's alpha coefficient for subscales was reported from 0.69 to 0.87 , and Cronbach's alpha coefficient of the whole questionnaire was 0.80 . The questionnaire scores range from 24 to 126 ( 24 to 42 - mild care burden, 49 to 71 - moderate, 72 to 95 - severe, 96 to 126 - very severe care burden) $[24,25]$. The content validity index of the questionnaire was reported as $91.31 \%$, and the reliability of the questionnaire using Cronbach's alpha coefficient was reported in Iran as $0.90[26]$.

\section{Statistical analysis}

Data was analyzed by SPSS version 24 software, inferential statistics (Chi-square, Fisher's exact test, independent $t$-Test, Mann-Whitney and repeated measures ANOVA), descriptive statistics (mean, standard deviation), central indicators and dispersion.

\section{Ethical approval}

The study conformed to the principles for ethical research contained in the Declaration of Helsinki (World Medical Association, 2013). Ethical approval was obtained from the ethics committee at Baqiyatallah University of Medical Sciences, Tehran, Iran (Code number: IR.BMSU.REC.1396.560). Voluntary participation was ensured. Written informed consent was obtained from all participants, and the information was kept confidential. This research was submitted in the form of a master thesis in nursing on IRCT systems (with Code number: IRCT20130611013636N2).

\section{Results}

In total, $51.4 \%$ of the sample were female and $48.6 \%$ were male. Fisher's exact test did not show any difference between the two groups in terms of gender $(p=0.5)$. There was no statistically significant difference between the two groups in the type of education ( $p=0.59$ ) (Table 2 ). The paired $t$-Test showed that there was no statistically significant difference in the control group before and after the study $(p=0.75)$ (Table 3$)$, while the paired $t$-Test showed that there was a statistically significant difference in the intervention group before and after the study $(p<0.001)$ (Table 4).

\begin{tabular}{|l|l|l|l|}
\hline \multicolumn{3}{|l|}{ Table 2. Demographic characteristics in the control and intervention groups in terms of gender and education } \\
\hline $\begin{array}{l}\text { Group } \rightarrow \\
\text { Dimensions } \downarrow\end{array}$ & Control number (\%) & Intervention number (\%) & Statistical test \\
\hline Male & $17(47.2)$ & $18(50)$ & $\begin{array}{l}\text { Fisher's exact test }=0.056 \\
p=0.5\end{array}$ \\
\hline Female & $19(52.8)$ & $18(50)$ & $\begin{array}{l}X^{2}=1.09 \\
p=0.95\end{array}$ \\
\hline Academic education & $22(61.1)$ & $18(50)$ & $18(50)$ \\
\hline Non-academic education & $14(38.9)$ & 18 &
\end{tabular}

\begin{tabular}{|c|c|c|c|}
\hline $\begin{array}{l}\text { Control Group } \rightarrow \\
\text { Dimensions } \downarrow\end{array}$ & $\begin{array}{l}\text { Before intervention } \\
\text { Mean (SD) }\end{array}$ & $\begin{array}{l}\text { After intervention } \\
\text { Mean (SD) }\end{array}$ & Paired $t$-Test \\
\hline Time dependent & $18.8(4.8)$ & $18.5(4.6)$ & $\begin{array}{l}t=0.89 \\
p=0.38\end{array}$ \\
\hline Evolutionary & $15.5(5.9)$ & $15.5(6.1)$ & $\begin{array}{l}t=-0.55 \\
p=0.95\end{array}$ \\
\hline Physical & $10.8(4.4)$ & $6.61(1.81)$ & $\begin{array}{l}t=5.53 \\
p<0.001\end{array}$ \\
\hline Social & $8.9(3.9)$ & $9.6(4.9)$ & $\begin{array}{l}t=-1.15 \\
p=0.25\end{array}$ \\
\hline Emotional & $11.6(4.6)$ & $10.8(4.1)$ & $\begin{array}{l}t=1.32 \\
p=0.19\end{array}$ \\
\hline Total score & $65.9(18.4)$ & $65.4(18.8)$ & $\begin{array}{l}t=0.317 \\
p=0.75\end{array}$ \\
\hline
\end{tabular}

\begin{tabular}{|l|l|l|l|}
\hline \multicolumn{3}{|l|}{ Table 4. Comparison of the mean and standard deviation of the care burden in the intervention group before and after intervention } \\
\hline $\begin{array}{l}\text { Intervention Group } \rightarrow \\
\text { Dimensions } \downarrow\end{array}$ & $\begin{array}{l}\text { Before intervention } \\
\text { Mean (SD) }\end{array}$ & $\begin{array}{l}\text { After intervention } \\
\text { Mean (SD) }\end{array}$ & Paired $t$-test \\
\hline Time dependent & $20.3(4.6)$ & $16.6(3.58)$ & $\begin{array}{l}t=1.82 \\
p=0.07\end{array}$ \\
\hline Evolutionary & $13.6(5.5)$ & $11(4)$ & $\begin{array}{l}t=5.03 \\
p<0.001\end{array}$ \\
\hline
\end{tabular}




\begin{tabular}{|l|l|l|l|}
\hline \multicolumn{4}{|l|}{ Table 4. Comparison of the mean and standard deviation of the care burden in the intervention group before and after intervention } \\
\hline $\begin{array}{l}\text { Intervention Group } \rightarrow \\
\text { Dimensions } \downarrow\end{array}$ & $\begin{array}{l}\text { Before intervention } \\
\text { Mean (SD) }\end{array}$ & $\begin{array}{l}\text { After intervention } \\
\text { Mean (SD) }\end{array}$ & Paired $t$-test \\
\hline Physical & $11.3(3.9)$ & $6.2(1.7)$ & $\begin{array}{l}t=8.22 \\
p<0.001\end{array}$ \\
\hline Social & $8.1(3)$ & $6.6(1.7)$ & $\begin{array}{l}t=3.89 \\
p<0.001\end{array}$ \\
\hline Emotional & $11.6(4.9)$ & $9.4(3.4)$ & $\begin{array}{l}t=5.56 \\
p<0.001\end{array}$ \\
\hline Total score & $65.2(17.1)$ & $53.1(13.6)$ & $\begin{array}{l}t=6.79 \\
p<0.001\end{array}$ \\
\hline
\end{tabular}

\section{Discussion}

Spiritual care based on the Sound Heart Model was effective in reducing the parents' care burden. The model considers the spiritual caregivers as mentors, with an emphasis on spiritual communication, having a friendly behavior with empathy and respecting the beliefs and culture of the patients and families, as well as treating the patients in accordance with ethical standards [27]. It introduces communication as the first step of empowerment and an important factor in gaining trust, reducing the stress and anxiety of patients, and emphasizes the parents' spiritual self-care [28]. This research was done based on a care model, because models, as functional guidance, can facilitating home care and the familiarity of the treatment team, with care models is essential [29] but unfortunately, the use of care models in Iranian clinical services is limited [30]. In spiritual care sessions with family-centered approach for reducing parental distress, religious evidence-based guidelines for emotional adaptation were taught, because most caregivers suffer from high levels of care burden and depression and do not use proper coping strategies, which lead to family dysfunction [31].

Although Steele and et al. reported that the stress and emotional distress of mothers of cancerous children has decreased over the course of 6 months though their care burden remains unchanged [32], in this study, spiritual care was able to reduce the care burden of the intervention group after 4 months. This seems to be due to attention to the impact of spirituality and religious beliefs in creating patience, hope in God's mercy and optimism for the future and finding a meaning for life events. In this study, the parents' care burden was moderate, which is consistent with the results of the Ahmadi et al. study [33], but it is not in alignment with the research by Valizadeh et al. that reported high levels of care burden in 150 parents in Tabriz [34]. Chang et al. reported that caregivers who have to spend more time on patient care due to the severity of the patient's condition and care needs are exposed to higher care burden and had lower mental health [35]. Care burden of a personal nature is also related to the caregiver status and the level of their moral development [36]. Thus, in counseling sessions, parents' attention was drawn to the positive and hidden aspects of unpleasant life events. By advising them to leave their problems to God, to increase optimism about receiving divine grace and love, to attract God's love by loving needy people and to have compassion for the creatures of the world, an attempt was made to strengthen the moral virtues in parents. By supporting parents in weekly telephone calls, efforts were also made to replicate "software training" and encourage its implementation, which was consistent with the study by Chung and Hwang about the effectiveness of self-care and home-based education by telephone on the quality of life of 70 leukemic patients undergoing chemotherapy [37]. Due to the shortage of nursing staff in Iran, it is recommended that follow-up education be provided through special educational software for each patient group and through a social network.

\section{Limitations of the study}

Spiritual care is expected to be provided by a team of experts, including a physician, nurse, social worker, clinical psychologist and clergyman, with the collaboration of the patients and families, but in this research, only a nursing student, with the help of educational software and the social network, taught spiritual skills.

\section{Conclusions}

The findings of this study showed that spiritual care based on the Sound Heart Model decreased the level of care burden of parents, and thus the use of the model is suggested.

Acknowledgments. We would like to sincerely thank the Deputy of Research and Technology (Clinical Research Development Unit) of Baqiyatallah hospital for his support and advice, as well as the Pediatrics staff of the Children's Medical Center and all the families who collaborated in conducting this study.

Source of funding: This work was supported through the financial assistance of the Medicine Quran and Hadith research center of Baqiyatallah University of Medical Sciences.

Conflicts of interest: The authors declare no conflicts of interest.

\section{References}

1. Mohammadi MR, Ahmadi N, Kamali K, et al. Epidemiology of psychiatric disorders in Iranian children and adolescents (IRCAP) and its relationship with social capital, life style and parents' personality disorders: study protocol. Iran J Psychiatry 2017; 12(1): 66-72.

2. Rahimi S, Fadakar Soghe R, Tabri R, et al. Related factors with quality of life among preschool children with cancer. J Holist Nurs Midwifery 2014; 24(1): 30-39.

3. Mojen LK, Rassouli M, Eshghi P, et al. Pediatric palliative care in Iran: applying regionalization of health care systems. Asian Pac J Cancer Prev 2018; 19(5): 1303, doi: 10.22034/APJCP.2018.19.5.1303.

4. Pelland-Marcotte M-C, Hwee J, Pole JD, et al. Incidence of infections after therapy completion in children with acute lymphoblastic leukemia or acute myeloid leukemia: a systematic review of the literature. J Leuk Lymphoma 2019; 60(9): 2104-2114, doi: $10.1080 / 10428194$. 
5. Rassouli M, Salmani N, Mandegari Z, et al. Challenges of palliative care for children with cancer in Iran: a review. Iran J Ped Hematol Oncol 2019; 9(1): 48-62.

6. Cheraghi F, Feizy Barnaji A, Tapak L, et al. The relationship between general health status and quality of life of parents and quality of life of children with cancer. Avicenna J Nurs Midwifery Care 2018; 25(5): 200-207.

7. McCorkle R, Ercolano E, Lazenby M, et al. Self-management: enabling and empowering patients living with cancer as a chronic illness. CA Cancer J Clin 2011; 61(1): 50-62, doi: 10.3322/caac.20093.

8. Phillips F, Prezio EA. Wonders \& Worries: evaluation of a child centered psychosocial intervention for families who have a parent/primary caregiver with cancer. Psychooncology 2017; 26(7): 1006-1012, doi: 10.1002/pon.4120.

9. Aliakbarzadeh-Arani Z, Taghavi T, Sharifi N, et al. Impact of training on social adaptation in mothers of educable mentally-retarded children based on Roy adaptation theory. Feyz 2012; 16(2): 128-134.

10. Papastavrou E, Charalambous A, Tsangari H. How do informal caregivers of patients with cancer cope: a descriptive study of the coping strategies employed. Eur J Oncol Nurs 2012; 16(3): 258-263, doi: 10.1016/j.ejon.2011.06.001.

11. Williams PD, Williams KA, Williams AR. Parental caregiving of children with cancer and family impact, economic burden. Issues Compr Pediatr Nurs 2014; 37(1): 39-60, doi: 10.3109/01460862.2013.855843.

12. Rafiyah I. Review: burden on family caregivers caring for patients with schizophrenia and its related factors. NMJN 2011; 1(1): 29-41, doi: 10.14710/nmjn.v1i1.745.

13. Gold Zweig G, Merims S, Ganon R, et al. Informal caregiving to older cancer patients: preliminary research outcomes and implications. Ann Oncol 2013; 24(10): 2635-2640, doi: 10.1093/announce/mdt250.

14. Rosenberg AR, Dussel V, Kang T, et al. Psychological distress in parents of children with advanced cancer. JAMA Pediatr 2013; 167(6): 537-543, doi: 10.1001/jamapediatrics.2013.628.

15. Arief YS, Nursalam N, Ugrasena IDG, et al. Family factors that affect family empowerment in caring children with leukemia. Health Notions 2018; 2(4): 457-459.

16. Rad GHM, Mahmoud FK, Nemati A. Evaluation of the effects of the family-centered empowerment model on the nutrition style and physical activity of patients with asthma. Med Surg Nursing J 2016; 5(2): 35-41.

17. Hassankhani H, Taleghani F, Mills J, et al. Being hopeful and continuing to move ahead: religious coping in Iranian chemical warfare poisoned veterans, a qualitative study. J Relig Health 2011; 49(3): 311-321, doi: 10.1007/s10943-009-9252-z.

18. Abolghasemi $\mathrm{H}$, Asadzandi M. Reinforcing faith, the main care method of maintaining and improving the spiritual health of patients and clients. IJHP 2018; 1(1): 39-49.

19. Akbarpour H, Asadzandi M, Saffari M, et al. Effect of spiritual care based on Sound-Heart Consulting Model (SHCM) on spiritual health of hemodialysis patients. Crit Care Nursing J 2017; 10(4): 1-6.

20. Asadzandi M. Sound heart: Spiritual Nursing Care Model from religious viewpoint. J Relig Health 2017; 56: 2063-2075, doi: 10.1007/ s10943-015-0038-1.

21. Asadzandi M. An Islamic Religious Spiritual Health Training Model for patients. J Relig Health 2020 59: 173-187, doi: 10.1007/s10943018-0709-9.

22. Zafarian Moghaddam E, Behnam Vashani HR, Reihani T, et al. The Effect of spiritual education on depression, anxiety and stress of caregivers of children with leukemia. JMS 2016; 4(1): 1-7.

23. Asadzandi M. Guide book of spiritual care for Patients' family. Tehran: Resaneh-takhassosi; 2018.

24. McCrery A, Addington J, Addington D. Family assessment in early psychosis. Psychiatry Res 2007; 152(2-3): 95-102, doi: 10.1016/j. psychres.2006.07.002.

25. Novak M, Guest C. Application of a multidimensional caregiver burden inventory. Gerontologist 1989; 29(6): 798-803, doi: 10.1093/ geront/29.6.798.

26. Abbasi $\mathrm{A}$, Shamsizadeh $\mathrm{M}$, Asayesh $\mathrm{H}$, et al. The relationship between caregiver burdens with coping strategies in Family caregivers of cancer patients. IJPN 2013; 1(3): 62-71.

27. Asadzandi M. Principles of spiritual communication based on religious evidence in the "Sound Heart Model". J Med Therapeutics 2018; 2(3): $1-5$.

28. Asadzandi M, Shahrabi Farahany S, Abolghasemy H, et al. Effect of Sound Heart Model - based spiritual counseling on stress, anxiety and depression of parents of children with cancer. Iran J Ped Hematol Oncol 2020; 10(2): 96-106.

29. Khanali Mojen L, Rassouli M, Eshghi P, et al. Pediatric palliative care in Iran: applying regionalization of health care systems. Asian Pac J Cancer Prev 2018; 19(5): 1303-1311, doi: 10.22034/APJCP.2018.19.5.1303.

30. Caruso Brown AE, Howard SC, Baker JN, et al. Reported availability and gaps of pediatric palliative care in low-and middle-income countries: a systematic review of published data. J Palliative Med 2014; 17(12): 1369-1383, doi: 10.1089/jpm.2014.0095.

31. Arshi M, Gholami Jam F, Nazif A, et al. The relationship between caregiving burden and family function of children with cancer. Social Work Mag 2016; 4(4): 35-41.

32. Steele RG, Long A, Reddy KA, et al. Changes in maternal distress and child-rearing strategies across treatment for pediatric cancer. J Pediatr Psychol 2003; 28(7): 447-452, doi: 10.1093/jpepsy/jsg035.

33. Ahmadi M, Rassouli M, Karami M, et al. Care burden and its related factors in parents of children with cancer. IJN 2018; 31(111): 40-51.

34. Valizadeh L, Joonbakhsh F, Pashaee S. Determinants of care giving burden in parents of child with cancer at Tabriz children medical and training center. J Clin Nurs Midwifery 2014; 3(2): 13-20.

35. Chang H-Y, Chiou C-J, Chen N-S. Impact of mental health and caregiver burden on family caregivers' physical health. Arch Gerontol Geriatr 2010; 50(3): 267-271, doi: 10.1016/j.archger.2009.04.006.

36. Chung Y-C, Hwang H-L. Education for homecare patients with leukemia following a cycle of chemotherapy: an exploratory pilot study. Oncol Nurs Forum 2008; 35(5): 83, doi: 10.1188/08.ONF.E83-E89.

37. Lee K, Martin P, Poon LW. Predictors of caregiving burden: impact of subjective health, negative affect, and loneliness of octogenarians and centenarians. Aging Ment Health 2016: 1-8, doi: 10.1080/13607863.2016.1206512.

Tables: 4

Figures: 0

References: 37

Received: 15.12 .2020

Reviewed: 27.12 .2020

Accepted: 13.03.2021
Address for correspondence:

Minoo Asadzandi, PhD

Vanak Square

South Sheikh Baha'i, Nosrati Alley

Baqiyatallah University of Medical Sciences

P.O. Box: 19575-174, Tehran, Iran

Tel.: 09123769064

E-mail: mazandi498@gmail.com 